\title{
THE DESIGN OF AN INTERACTIVE E-LEARNING PLATFORM FOR SURVEYING EXERCISE
}

\author{
Shu-Chen Cheng ${ }^{\mathrm{a}, *}$, Peter T.Y. Shih ${ }^{\mathrm{b}}$, Shing-Lin Chang ${ }^{\mathrm{a}}$, Guan-Yu Chen ${ }^{\mathrm{a}}$ \\ ${ }^{a}$ Department of Computer Science and Information Engineering, Southern Taiwan University, Tainan, Taiwan - \\ kittyc@mail.stut.edu.tw \\ b Department of Civil Engineering, National Chiao-Tung University, Hsinchu, Taiwan - tyshih@mail.nctu.edu.tw
}

KEY WORDS: E-learning, M-learning, E-portfolio, Surveying exercise, Peer assessment, Collaborative learning, Cooperative learning, Dual-coding theory

\begin{abstract}
:
Surveying exercise is a fundamental course for Civil Engineering students. This course is featured with field operation. This study explores the design of an e-learning platform for the surveying exercise course. The issues on organizing digital contents such as recorded video of the standard instrument operation, editing learning materials, and constructing the portfolio for the learning process, as well as generating learning motivation, are discussed. Noting the uploaded videos, publishing articles and commentaries, interactive examination sessions, assessing for each other, and mobile device accessing, are found to be useful elements for this platform.
\end{abstract}

\section{INTRODUCTION}

In civil engineering constructions, site investigation prior to starting construction, engineering control whilst under construction, and building certification after construction all depend on the surveying. Therefore, surveying is a fundamental course for civil engineering. Surveying consists of the surveying method, surveying instruments, and correction of calculations. Traditionally, the Surveying course consists of theoretical teaching and practical surveying exercises. The theoretical teaching involves teaching students various surveying methods and correction of calculations in the classroom; and the practical surveying exercises teach students various operations of surveying instruments or usages of tools outside of the classroom, including practical operation in the field.

Traditional teaching for practical surveying exercises involves the teacher gathering students in an appropriate outdoor location to demonstrate the operation of objective instruments or usage of tools, explaining the operational flowchart and critical points when performing the surveying. Students are divided into several terms per 5-6 students. These terms are dispersed to independently practice the items that teacher instructs. Finally, the teams bring back the collected data to the classroom for relative corrections of calculations. Students can learn the operation of instruments and correct surveying flowcharts from the proper instruction of the teacher, and precise operation which is the same as team work as part of an actual surveying team. However, there are some disadvantages in this kind of teaching method such as students don't find it easy to pay attention to either what they should see or listen to in outdoor demonstrations or focus on operational demonstration. Meanwhile, the teacher may also find it difficult to monitor every situation of all teams in a wide range field operation. In case of any emergency, the teacher may not be able to take control in time. In addition, expensive and rare surveying instruments may cause students not to conduct previews before class and reviews after class.
This study intends to design an e-learning system to assist traditional surveying exercise classes and improve its deficiencies. The system is designed that the teachers can edit or upload learning materials, and provides the functions of tests. Therefore, students can do preview and review. Dual-coding theory is applied for both verbal and multi-media learning material, which can receive multiple sources and deepen understanding and memory of the students. The teaching method using operational team exercises achieves the cooperative learning. Using the system, students can share themselves learning achievements, and review the results or contents from others. Furthermore, team members can assess, criticize, make suggestions or provide feedback among them according to peer assessment theory, which makes up the teacher's overall consideration of all teams and individual performance.

The system provides mobile device access so that students can download learning materials or demonstration videos to smart mobile phones before class, or for use as operational instruction for practical exercises. They also can take photos and videos for themselves or team members, share them immediately, and appreciation and evaluation with others in the system. This system expects to offer various easy functions related to the entire course and learning process to really assist the teaching or learning.

\section{LITERATURE REVIEW}

\subsection{Surveying exercise}

Surveying is a fundamental course for Civil Engineering. Traditionally, surveying is defined as a science that studies the shape and size of the earth and assures the coordinates and relations of points on the ground. Nowadays, following the progress of instruments and technology, as well as expansion of study scope, surveying is re-defined as a science that studies the 
shape, size, direction, location, and distribution of each object in a 3-dimensional space. Surveying consists of two parts: measurement and location. Measurement refers to the use of surveying instruments and tools in surveying methods and calculations to acquire ground location data or topographic maps. Location refers to the mark on-the-spot by dividing the planned and designed structure or boundary on the blueprint as the basis of engineering construction or land delimitation. Location is also called Marking (Ghilani, Wolf, 2011).

Surveying depends on the combined use of instruments, methods and calculations, and as such the surveying course is always taught together with practical surveying exercises. Practical surveying exercises enable the student surveyor to practice and become familiar with flowchart and operational methods for various surveying instruments or tools. Practical exercises provide practice in various surveying methods and corrections of calculation, such as Levelling survey, Trigonometric levelling or Topographic map surveying, etc (Ghilani, Wolf, 2011).

\subsection{Cooperative learning}

Cooperative learning is a teaching strategy using teams to cooperate with each other in a systematic and organizational approach. After class, the teacher divides the students into heterogeneity groups of 4-6 students based on capability, gender, race, and background while students respectively conduct cooperative learning in each team to attain common personal and team performance goals, and to enhance the learning effect (Slavin, 1995, Johnson \& Johnson, 1999).

Cooperative learning features positive mutual interdependence, face to face interaction, emphasis of personal performance and responsibility, development of interpersonal relationships, team work, group experience and team achievement among students. Cooperative learning trains students to mutually depend on and proactively cooperate among themselves, thus further increasing the learning effect (Johnson \& Johnson, 1999).

The advantages of cooperative learning implementation are to promote high quality development of cognitive strategies, to enhance the learning motivation of students, to improve academic performance, and to encourage active learning. It can lead to conflict of ideas between students, and to increase the awareness and respect of students to multi-cultural, so that the learning experience from students with different backgrounds to exchange. And to enhance the language skills of students, i.e. listening, speaking, reading and writing skills, enhance the social skills of students, and enhance the performance of teachers (Slavin, 1995, Johnson \& Johnson, 1999).

In recent years, the progress and popularity of personal computers and internet technology covering handheld mobile devices has expanded cooperative learning from traditional classrooms to the worldwide internet. Interactive and small group learning through computer-aided technology, software or websites facilitate discussions on relevant problems experienced by team members without the limitation of time and space to boost the enjoyment of learning over the internet and complete the learning goals on both a personal and team level. Cooperative learning will be an important trend for the development of an internet digital learning environment (Lin, 2010).

\subsection{Peer assessment}

Peers are classmates who have similar maturity of knowledge, professional techniques, learning backgrounds, or similar age and position. Peer assessment is a kind of learning model, in which learners role play as teachers in a learning situation that simulates a peer environment to assess, compare, criticize or suggest to the meanings, quantities, qualities, applications, values or successes for mutual learning results or work (Topping, 1998).

Learners in the peers assessment should offer others feedback, suggestions, questions or criticism, and point out the deficiencies of others in the process. The assessor who receives peer feedback can improve or correct ones work. Similar learning experiences or capability backgrounds between peers and the assessor can facilitate better understanding about problems and difficulties on the same operation or items. Therefore, sufficient information and specific viewpoints can highlight what teachers might miss (Topping, Ehly, 2001).

\subsection{Dual-coding theory}

Dual-coding theory points out that people possess two sets of interactive but independent systems that deal with different kinds of information, i.e. Logoens and Imagens. After Logoens receive a verbal stimulation, it will represent these information, and store the codes in the verbal memory zone. Imagens specifically deals with visual stimulations. After representing these visual information, it will store the codes in the visual memory zone, and leave a verbal contrast version against the verbal memory zone. There is a connection between Logoens and Imagens, i.e. Referential connection (Paivio 1986).

When humans learn, their working memory has three processes for the presentation of information as either verbal or image. Working memory receiving verbal stimulation will build an image with a verbal description. This recognition process from the exterior to interior is called building a verbal representational connection or verbal encoding. Working memory receiving visual stimulation will build an image with a visual description. This recognition process from the exterior to interior is called building a visual representational connections or visual encoding. Referential connection connects two presentations of information while the learning effect depends on the quality established by verbal encoding, visual encoding, and referential connection. Therefore, the dual-coding theory view is that learners need to simultaneously use verbal encoding and visual encoding to effectively deal with information and promote the establishment of referential connection to improve the learning effect (Paivio, 1986, Clark, Paivio, 1991).

\subsection{M-learning}

Mobile learning (M-learning) refers to learners using hand-held mobile devices to download or transport learning contents through Wi-Fi or 3G to PDAs, Smart Phones or Tablet PCs for learning. Learning contents could consist of words, images, voice or even videos. Strong function and convenient portable mobile devices can help learners eliminate the limitation of crossing time and space. Mobile devices will be sharp weapons for acquiring knowledge or communication anytime and anywhere (Jeng et al., 2010, Huang et al., 2010, Huang et al., 2010b). 
E-learning was available before m-learning. E-learning refers to acquiring digital learning materials through the internet by using a PC. Learners who use e-learning can positively control their learning schedule and process without limitations of time and space. Above all, e-learning possesses a significant amount of interactive learning contents. However, m-learning uses Smart Phones or Tablet PCs, and other mobile devices to create an environment, which learners can get learning contents or communication anytime and anywhere, through the Universal Mobile Telecommunications System (UMTS). Figure 1 represents learners using mobile devices to interact with a learning platform through mobile or wireless network (Jeng et al., 2010, Huang et al., 2010, Huang et al., 2009).

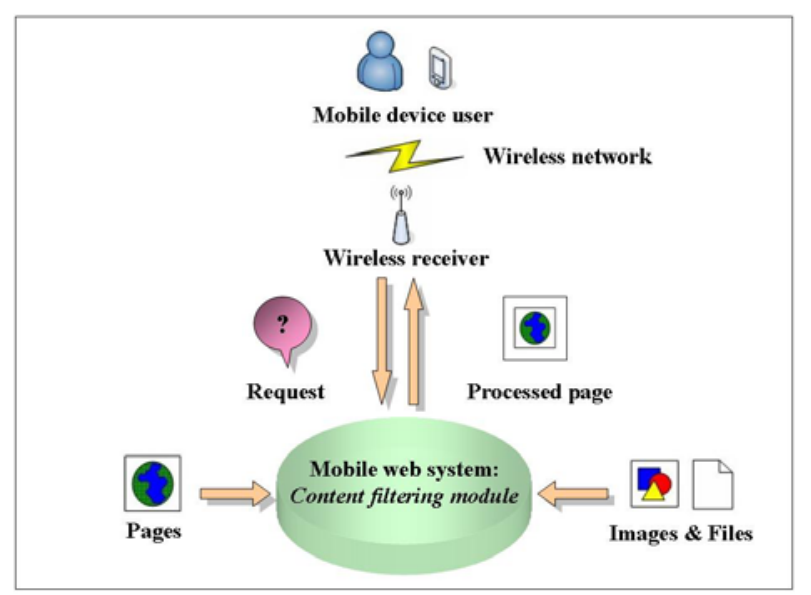

Figure 1. Mobile network diagram

The concept of m-learning does not intend to replace the PC with a mobile device, rather it is about using mobile devices to expand the functionality of the PC or eliminate the hindrance of a fixed installation. With excellent portable and immediate advantages, mobile devices can be used to learn anywhere. However, smaller screen size, lower calculation capability and inferior quality are problems that are inherent in mobile devices. The mobile device is not comparable with the PC in terms of the same amount of content, visual effect or interactive operation. Comfortable reading and watching media on mobile devices is incomparable with a PC. Therefore, mobile learning does not refer to directly reading normal digital learning materials on mobile devices. Instead, learning contents for learners must be firstly taken and screened through the integration of an e-learning platform and the content management module before transporting them to the learning device of learners for further presentation (Jeng et al., 2010, Huang et al., 2010, Huang et al., 2010b).

\subsection{E-portfolio}

Learning portfolio first appeared in the Renaissance period. Artists recorded personal articles with paper rolls to present creation and development in different periods, and help to understand progress or change for personal creative portfolios. Not until the 1980s had learning portfolios been used for application in the field of educating as a new trend. Recently, many studies have pointed out that learning portfolios can effectively enhance learning efficiency. The features of learning portfolios are as follows: 1) be able to systematically and organizationally present real learning situations; 2) to have reviewing and reflecting functions in self-learning portfolios; 3) to stimulate the interactive and cooperative learning spirit between peers; 4) to encourage the positive creativity of learner; 5) to connect with teaching assessment to strongly meet the individual differences of learners (Chang, 2001, Chen et al., 2000).

Traditionally, significant costs were incurred in recording on paper rolls, and it was inconvenient to store and arrange portfolios. As a result, digitalizing learning has become the current trend. The limitations of the establishment of traditional portfolios such as the limitations of time and space will be eliminated by the use of integrated computer and internet technology through immediate and interactive internet, and the immediacy for the establishment, storage or maintenance of portfolios will be increased. Internet connection through database technology is helpful to record, arrange, search, analyze and present learning portfolios, providing sharing functions between students and teachers, and reviewing learning portfolios from others to increase opportunities of interaction and communication (Chang, 2001, Chen et al., 2000).

The learning portfolio can be applied to various different courses, in which important educating functions will be assessment tools. The learning portfolio collects all kinds of different information from learners, especially where immediacy and interaction of internet are used to collect information, which further reflects or presents the real learning performance, situation of progress, or trend of change for learners (Su et al., 2006, Chang, 2001, Chen et al., 2000).

\section{SYSTEM ARCHITECTURE}

This study has designed an e-learning system to assist the progress of traditional learning for the practical surveying exercise course. Vivid contents and interactive operations are provided to stimulate or promote the passion and willingness of students to learn traditional courses. The system provides teachers with a friendly interface to edit and manage a range of learning materials, such as text web pages, files, videos and images, etc. Teachers can manage items and hold tests to students. Students can log into the system to preview or review any material at any time, or attend tests per requirement. The contents produced in the learning process of editing or uploading can be open for relevant persons, and then start mutual assessing or suggesting. The system will record all detailed operations in the system carried out by students to produce a personal learning portfolio for each student, which displays learning results or use as a basis for grading and reinforcing capability.

The system includes 9 modules: Account module, Group module, Message module, Video notes module, Learning material module, Test module, Assessment module, Portfolio module, and Mobile access module. The architecture of the system modules are shown in Figure 2. 


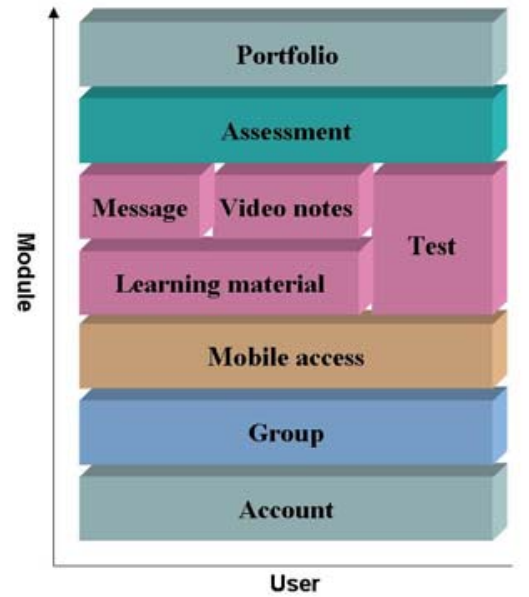

Figure 2. System architecture diagram

A detailed description for each module in the system is as follows:

Account module: This module includes two parts, Account Management and Role Management. Account Management deals with the relevant user accounts in the system, including adding accounts, amending account information, locking accounts and role assignment, etc. Role Management refers to manage the role of accounts. The System predefines three roles, Guest, Student, and Teacher. The authorization of each role is described in Table 1.

\begin{tabular}{cl}
\hline Role & \multicolumn{1}{c}{ Description } \\
\hline G u e s t & $\begin{array}{l}\text { Un-authorized user. Read or review } \\
\text { public learning material only. } \\
\text { Student }\end{array}$ \\
Authorized user. Be able to either \\
receive or post contents. \\
Teacher
\end{tabular}

Table 1. Description of role authorization

Group module: This module manages two kinds of groups in the system, Class and Team. The teacher can create one Class for each lecture, and add the accounts of students in. Establish several Teams for this Class, and then add students in each Team by the real grouping situation. Groups are the dependence of accessing the contents, such as learning materials or messages, etc., in the system.

Message module: In this module, the user can post text messages in the system, such as announcements, composition and commentary, etc., and can reply one certain posted text message. Posting and reading of text messages are controlled by roles and groups.

Video notes module: This module offers a friendly and easily operational interface that users can note on the uploaded video files, such as lines, arrows, circles, rectangles or text explanations, etc., and can edit captions for videos. Note can be stored independently. When the video is playing, the note will be played as like a mask at the same time. Therefore, different users can respectively make independent notes on the same video (Hwang et al., 2007, Mu, 2010).

Learning material module: This module is a tool used to edit and manage learning materials. The teacher can use this module to deal with three kinds of learning materials, text web pages, files, and videos. A friendly on-line editor is provided "What you get is what you see" to produce a text web pages in well format; offer a upload tool for files to deal with documents, images or videos and other files. Videos can be handled using the Video notes module after being uploaded for more vivid contents. Dual-coding theory points out learners can receive two or more information sources to effectively enhance the learning effect. This module offers several kinds of editing methods for learning materials, which is expected to provide advantages that can enhance the learning effect.

Each learning material item can be assigned to any group, in which the user belongs can see learning material for their own groups. In fact, all contents in the system are bound to groups. Whether learning materials or contents that students post is limited to viewing or acquiring by the members who belong to the assigned groups. Public learning materials or information must be assigned to a built-in special public group. Therefore, different classes can be assigned different learning materials.

If a video is assigned as learning material for a certain group, it can be set as two kinds of modes: normal and course. If the video is set as normal mode, its behaviours will just like a normal on-line video. If the video is set as course mode, it could only be played or paused. The system forces students to watch the video from the beginning to the end at least once, hereafter, this video becomes to normal mode that students can watch again or review at any time.

Test module: This module is used to manage test items, manage and produce test papers, manage and hold tests. The test items could be single-choice, multiple-choice, fill in the blank, Q \& A, or interactive test items in diagrams. The test paper could be manually checked, test ranges could be set. The number of test items, distribution of item types, and difficulty could be produced by the module automatically. Test papers can be stored and amended after being produced, but cannot be amended again after test has been held.

The test includes tests that students can ask for and teachers can hold. The tests that students ask for are mainly used to exercise the test system or review course contents, while teachers score tests that are held. The test could be held in a fixed time and period, e.g. ask students to do the test in the classroom; could be in a fixed time but not period, e.g. ask students to log into the system for the test within a certain period. Test papers with the same test items can be used for the test in the same period, or the system can randomly produce test papers for students according to parameters set.

When a teacher edits a video learning material, he can choose several test items to bind to that video. After students watch this video, they will be requested by the system to answer these items for the purpose of reminder or review.

Assessment module: This module processes assessments for the system contents. Cooperative learning makes learners in groups learn in an environment of mutual cooperation, stimulation, interaction, and feedback. Furthermore, peer assessment makes learners in the team mutually assess the results to attain the purpose of reconsideration through communication and feedback. This module is designed on the basis of cooperative learning and peer assessment. Students can take photos or shoot videos for their own learning processes or results, and then upload them to the system or post composition or commentary to share with others. Teachers can shoot videos 
for the process or operational exercises to post onto the system. Students can assess or comment on this content, the result of which can provide teachers with references or sources of the final scoring. Figure 3 shows the diagram for the whole process from grouping, learning, assessing to final scoring by the system (Chang, Chen, 2009, Li, Steckelberg, 2005, Tseng, Tsai, 2007).

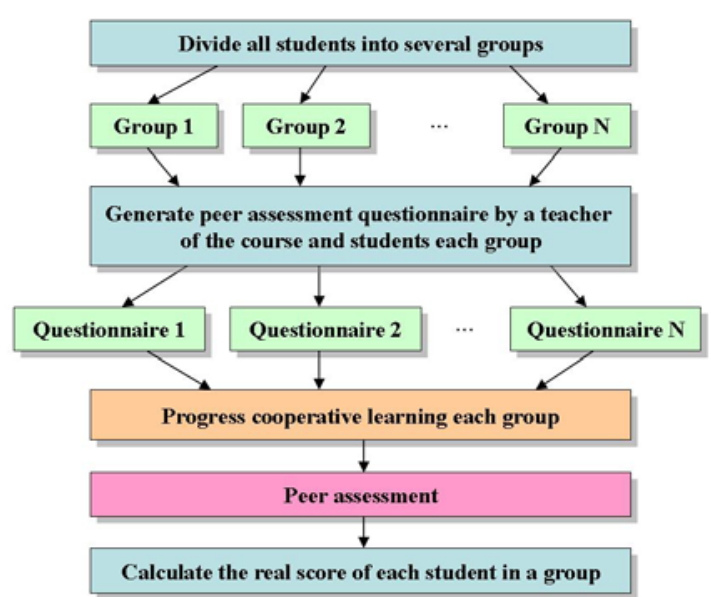

Figure 3. The flow of grouping and assessment

Portfolio module: This module is a record module mainly called by other modules, recording a detailed portfolio that users have used the system. Finally, the portfolio for the usage of the system by each user will be produced to present learning results, by which teachers or students can adjust or reinforce their learning situations. All portfolio that users produce are valuable and important, which can be used to analyze the arrangement of course contents or system design for further improvement (Su et al., 2006, Chang, 2001).

Mobile access module: This module refers to the use mobile devices, such as PDAs, Smart Phones or Tablet PCs, etc. to connect the system to deal with the contents presented or responded to. Since mobile devices have insufficient calculation capabilities issues, smaller screen size, narrower broadband width and unstable quality for network, etc. the presentation of content is not the same as or isn't available for the visual effect of the PC. Therefore, this module will delete unnecessary effects of the image, layout or JavaScript to show users necessary content by reducing transportation quantity and calculation demand (Huang et al., 2010, Huang et al., 2010b).

At present, Smart Phones and Tablet PCs gain popularity. Some demonstration video files can be downloaded or immediately transported to the devices for operation process, which can be presented during a field exercise. The operation videos of students can be shot to upload to the server, and teachers or teaching assistants can use the note functions of the videos to either score or correct mistakes accordingly. Students also can review the videos by themselves, and peers can observe or mutually assess. Therefore, mobile devices can replace PCs to become a full-functional simple version (Huang et al., 2009).

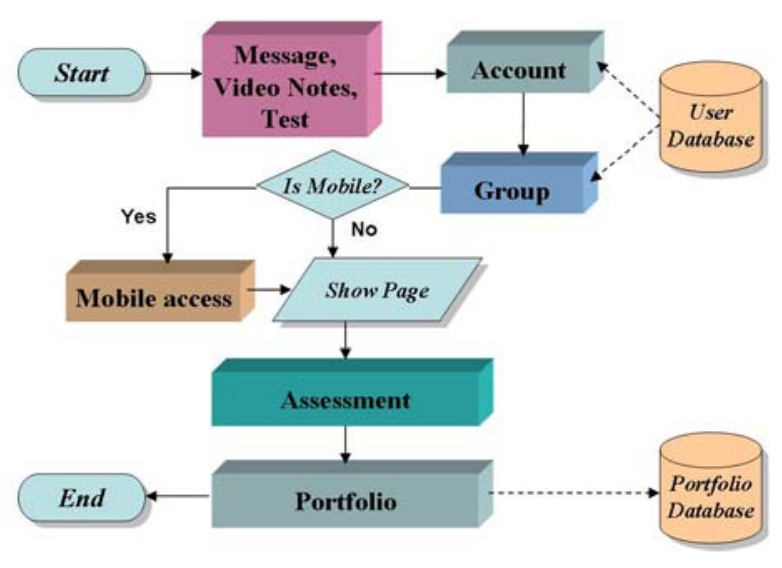

Figure 4. Module interactive diagram

Each module connects to each other, starting from logging in the system by the Account module to confirming authorization by the Group module, and finally the Portfolio module which is the rest of the functional modules records the portfolio. The mutual interactive relationships are shown in Figure 4.

\section{DISCUSSION AND CONCLUSION}

\subsection{System evaluation}

According to the concept of cooperative learning, heterogeneous grouping is normally supposed to be the best one for learning effect. However, team members with certain coordination and mutual understanding are more realistic in a survey team in actual surveying, and thus homogeneous grouping is preferable. Therefore, it is necessary to analyze how the learning effect differs according to grouping in terms of this system. This study is expected to collect data from three different grouping classes: First, traditional method, students conduct grouping by themselves without using the system, which will automatically become homogeneous grouping; Second, students conduct grouping by themselves but use the system to assist teaching; Third, teachers decide to conduct heterogeneous grouping for students. In this way, we can compare the change of learning situation in each team between using the system and heterogeneous or homogeneous grouping, and assess the assistant effect of this system.

\subsection{User portfolio}

The system will record in detail log in time and usage duration of each account, as well as every operation of the user, such as composition posted, commentary posted, videos shared, videos watched, assess other and process test, etc. to calculate the usage frequency and usage time of student, desire for discussion and assessing, comparing if a student who often uses this system has better performance in terms of the learning result.

Compare results from the analysis and final questionnaire to ensure that the actual operational actions of student accords with self-recognition, or has differences. Therefore, the results of questionnaires are used as a basis of improvement of this system. Comparison of the assessment results of student each time with teacher or teaching assistant results will indicate a stable progress learning effect is being achieved if the results of student gradually get closer to that of the teacher or teaching assistant. In contrast, if the results of student gradually drift 
further away from that of the teacher or teaching assistant, it means the learning effect of student has reduced. The teacher must pay special attention to learning situation and schedule of student.

Learning portfolio will represent the learning situation of a student in real time. If the learning effect has reduced, or if there are any deficiencies, the student can focus on improving on the weakness, or proactively ask for assistance from a teacher.

\subsection{Future work}

This study proposes an e-learning system to assist in the teaching of surveying exercises. It offers students some additional tools for communication and interaction besides real field operation team. This system still needs further improvement, for example, by adding a computerized adaptive test (CAT) module to increase the efficiency of testing. Considering expensive and rare surveying instruments, we should apply current popular 3D graphic technology to establish a $3 \mathrm{D}$ virtual model for each instrument, and then processing $3 \mathrm{D}$ virtual exercises in the system to really offer a preview function before class and review function after class.

\section{REFERENCES}

Chang, C.-C., 2001. Construction and Evaluation for a Webbased Learning Portfolio System: An Electronic Assessment Tool. Innovations in Education and Teaching International, 38(2), pp. 144-155.

Chang , T.-Y., Chen, Y.-T., 2009. Cooperative learning in Elearning: A peer assessment of student-centered using consistent fuzzy preference. Expert Systems with Applications, 36, pp. 8342-8349.

Chen, G.-D., Liu, C.-C., Ou, K.-L., Liu, B.-J., 2000. Discovering Decision Knowledge from Web Log Portfolio for Managing Classroom Processes by Applying Decision Tree and Data Cube Technology. Journal of Educational Computing Research, 23(3) pp. 305-332.

Cheng, I., Basu, A., 2009. Interactive Graphics for Computer Adaptive Testing. COMPUTER GRAPHICS forum, 28(8), pp. 2033-2045.

Clark, J. M., Paivio, A., 1991. Dual Coding Theory and Education. Educational Psychology Review, 3(3), pp. 149-210.

Ghilani, C. D., Wolf, P. R., 2011. Elementary Surveying: An Introduction to Geometrics, 13th Ed. Prentice Hall.

Huang, J. J.-S., Yang, S. J.-H., Huang, Y.-M., Hsiao, I. Y.-T., 2010. Social Learning Networks: Build Mobile Learning Networks Based on Collaborative Services. EDUCATIONAL TECHNOLOGY \& SOCIETY, 13(3), pp. 78-92.

Huang, Y.-M., Lin, Y.-T., Cheng, S.-C., 2010b. Effectiveness of a Mobile Plant Learning System in a science curriculum in Taiwanese elementary education. COMPUTERS \& EDUCATION, 54(1), pp. 47-58.
Huang, Y.-M., Yang, S. J.-H., Tsai, C.-C., 2009. Web 2.0 for interactive e-learning. INTERACTIVE LEARNING ENVIRONMENTS, 17(4), pp. 257-259.

Hwang, W.-Y., Wang, C.-Y., Sharples, M., 2007. A study of multimedia annotation of Web-based materials. Computers \& Education, 48, pp. 680-699.

Jeng, Y.-L., Wu, T.-T., Huang, Y.-M., Tan, Q., Yang, S. J.-H., 2010. The Add-on Impact of Mobile Applications in Learning Strategies: A Review Study. EDUCATIONAL TECHNOLOGY \& SOCIETY, 13(3), pp. 3-11.

Johnson, D. W., Johnson, R. T., 1999. Learning Together and Alone: Cooperative, Competitive, and Individualistic learning, 5th ed. Allyn and Bacon, MA.

Li, L., Steckelberg, A. L., 2005. Peer assessment support system (PASS). TechTrends, 49, pp. 80-85.

Lin, Y.-T., 2010. An Enhanced Particle Swarm Optimization Technique for Forming Collaborative Learning Groups Applied to a Web-based Learning System. Department of Engineering Science, NCKU.

Mu, X., 2010. Towards effective video annotation: An approach to automatically link notes with video content. Computers \& Education, xxx, pp. 1-12.

Paivio, A., 1986. Mental representations: a dual coding approach. Oxford, England: Oxford University Press.

Slavin, R. E., 1995. Cooperative learning: Theory, research, and practice, 2nd ed. Massachusetts, Allyn \& Bacon.

Su, J.-M., Tseng, S.-S., Wang, W., Weng, J.-F., Yang, J.-T. D., Tsai, W.-N., 2006. Learning Portfolio Analysis and Mining for SCORM Compliant Environment. Educational Technology \& Society, 9, pp. 262-275.

Topping, K. J., 1998. Peer Assessment between Students in Colleges and Universities. Review of Educational Research, 68(3), pp. 249-276.

Topping, K. J., Ehly, S. E., 2001. Peer-assisted learning. Journal of Educational and Psychological Consultation, 12(2), pp. 113-132.

Tseng, S.-C., Tsai, C.-C., 2007. On-line peer assessment and the role of the peer feedback: A study of high school computer course. Computers \& Education, 49(4), pp. 1161-1174.

\section{ACKNOWLEDGEMENTS}

This research was partially supported by the National Science Council, Taiwan, ROC, under Contract No.: NSC 97-2511-S218-003-MY3 and NSC 99-2511-S-218-007-MY2. 